\title{
Stratifying risk: asymptomatic carotid disease
}

\author{
Estratificação do risco na doença carotídea assintomática
}

Rafael D. Malgor', Emily A. Wood², Otavio A. lavarone 3 , Nicos Labropoulos ${ }^{4}$

\begin{abstract}
Stroke generates significant healthcare expenses and it is also a social and economic burden. The carotid artery atherosclerotic plaque instability is responsible for a third of all embolic strokes. The degree of stenosis has been deliberately used to justify carotid artery interventions in thousands of patients worldwide. However, the annual risk of stroke in asymptomatic carotid artery disease is low. Plaque morphology and its kinetics have gained ground to explain cerebrovascular and retinal embolic events. This review provides the readers with an insightful and critical analysis of the risk stratification of asymptomatic carotid artery disease in order to assist in selecting potential candidates for a carotid intervention.
\end{abstract}

Keywords: carotid artery diseases; risk; natural history.

\section{Resumo}

O acidente vascular encefálico gera custos significativos na área da saúde e representa um problema social e econômico. A instabilidade da placa carotídea aterosclerótica é responsável por um terço dos acidentes vasculares encefálicos embólicos. O grau de estenose tem sido usado para justificar, deliberadamente, intervenções carotídeas em milhares de pacientes no mundo todo. No entanto, o risco anual de acidente vascular encefálico em doença carotídea assintomática é baixo. A morfologia da placa e sua mobilidade têm ganhado importância na elucidação dos eventos embólicos cerebrovasculares e retinais. Esta revisão proporciona aos leitores uma análise crítica e inteligente da estratificação de risco da doença carotídea assintomática com o intuito de auxiliar na seleção de potenciais candidatos à intervenção carotídea.

Palavras-chave: doenças das artérias carótidas; risco; história natural.

\section{Introduction}

Stroke is responsible for significant healthcare costs and it is a social burden in Western societies ${ }^{1-3}$. Embolism is the most common cause of stroke, with carotid artery atherosclerotic plaques responsible for one in three embolic strokes. Clinical presentation of cerebrovascular and retinal embolic events varies from no symptoms to transient ischemic attack (TIA), amaurosis fugax (AF), and stroke. The asymptomatic carotid artery disease (ACAD) is an important cause of stroke accounting for about $8 \%$.

The Asymptomatic Carotid Atherosclerosis Study (ACAS) and the Asymptomatic Carotid Surgery Trial (ACST) found an annual risk of cerebrovascular events from 1 to $2 \%{ }^{4,5}$. Degree of stenosis was the only criterion used to indicate an intervention in both trials. In addition, medical treatment in the ACAS trial was significantly different from the previously recognized standards recommending 325 mg of aspirin coupled with educational advise about risk factors, such as diabetes, hypertension, and tobacco use ${ }^{4}$.

A systematic review of 3,724 patients with ACAD showed decreasing rates of stroke related to improvements in medical therapy ${ }^{6}$. A prospective cohort of 101 patients with ACAD also found lower annual risk of ipsilateral events $(\mathrm{AR}=0.34 \%, 95 \% \mathrm{CI} 0.01-1.87)$ when patients were treated with anti-platelet agents, statins, and anti-hypertensive medications ${ }^{7}$.

The critical question that remains unanswered is: who will benefit from an intervention versus a medical treatment alone? Risk stratification of patients with ACAD still relies

Study carried out at the Stony Brook Medical Center - New York, USA.

${ }^{1}$ Vascular Surgery Resident in the Division of Vascular Surgery at the Stony Brook Medical Center - New York, USA.

${ }^{2}$ General Surgery Resident in the Division of General Surgery at the Stony Brook Medical Center - New York, USA.

${ }^{3}$ Medical Student at Paulista School of Medicine - São Paulo (SP), Brazil.

${ }^{4}$ Professor of Surgery in the Division of Vascular Surgery at the Stony Brook Medical Center - New York, USA.

Financial support: none.

Conflicts of interest: nothing to declare.

Submitted on: 08.31.11. Accepted on: 09.25.11.

J Vasc Bras. 2012;11(1):43-52. 
upon degree of stenosis in the majority of vascular surgery practices. Also, the largest trials on carotid artery intervention only utilize the degree of stenosis to indicate a procedure ${ }^{4,5,8}$.

The concept of flow reduction to the brain causing tissue ischemia is simplistic and it must not be extrapolated to every case of ACAD. A subset analysis of 3,007 patients, who had a reduced vessel diameter distal to the area of stenosis, demonstrated a protective component resulting in low risk of ipsilateral stroke ${ }^{9}$. The former is the evidence that degree of stenosis alone should not always be considered in asymptomatic patients when making a decision regarding who would benefit from an intervention.

The role of plaque morphology and its instability causing either embolization or in situ thrombosis has been discussed ${ }^{10,11}$. Clinical features including cardiovascular risk factors (i.e., hypertension, hyperlipidemia) and life-style habits (i.e., smoking), coupled with plaque morphology and degree of stenosis, are important factors attributed to increased risk of disease progression and adverse outcomes, such as TIA and stroke.

\section{Natural history of asymptomatic carotid artery disease}

There are two different extremes of the same disease in symptomatic and asymptomatic patients with atherosclerotic carotid disease. Whilst the outcomes in the symptomatic group are poor if not treated in a timely fashion, the course of ACAD has a low event rate and therefore a much better prognosis. The natural history of ACAD has been extensively studied over the past three decades ${ }^{10,12-20}$.

ACAD patients are identified from the presence of cervical bruits, during the investigation of nonspecific signs and symptoms and prior to pre-operative evaluation for cardiac or other major surgery. The first imaging study utilized to rule out any cerebral ischemic events is a computed tomography (CT) scan of the head and a duplex ultrasound (DU) of the carotid artery. Evaluation with DU relies on determining the degree of carotid stenosis. The plaque characteristics are not routinely taken into account and are not reported on a standardized fashion.

Patients with abnormally raised DU velocities or ratios are stratified as having a mild, moderate, or severe stenosis. Accordingly, those patients are stratified and therefore offered a procedure or place on medical therapy if a non-high grade stenosis is found. After the publication of the ACAS in 1995, there was an increase in the accrual number of carotid endarterectomy (CEA) in the USA ${ }^{21,22}$. The number of asymptomatic patients treated, based on DU velocities, found in a national and statewide databases demonstrated that the majority of CEAs are performed to treat patients with $\mathrm{ACAD}^{23,24}$. The rationale of treating $\mathrm{ACAD}$ was the slightly lowest rate of cerebrovascular events in patients who underwent CEA in the ACST and ACAS, when compared to the Control Group receiving medical treatment. However, the stroke-free incidence found in the ACAS and ACST indicate that more than 94\% of the procedures were perhaps unnecessary ${ }^{25}$. Moreover, the patients enrolled in the ACST that were managed with best medical treatment (BMT) had risk of any or ipsilateral stroke of about 12 and $5 \%$ at a five-year period, which was reduced to 7 and $4 \%$ at the ten-year one.

Certain plaque morphology characteristics, such as ulceration and echolucent material, are known to have a more active cellular turnover making the plaque prone to rupture and to embolize ${ }^{26}$. Recent research on the plaque morphology and its components graded using grayscale median (GSM), white areas, and the area of the plaque have assisted in gauging the "benign" versus unstable plaques from cerebral and retinal ischemic embolic events ${ }^{10,27}$. The largest prospective study on patients with ACAD enrolled 1,121 consecutive patients as part of the Asymptomatic Carotid Stenosis and Risk of Stroke (ACSRS) Study Group ${ }^{10}$. All patients had DU assessment of the internal carotid artery (ICA) stenosis and plaque morphology, which were correlated to clinical characteristics and followed-up for a mean period of four years. The degree of stenosis of the ICA was found to be correlated with the outcomes. An increasing risk of ipsilateral cerebrovascular events was demonstrated in patients with severe stenosis (>90 to $99 \%$ European Carotid Surgery Trial - ECST criteria $^{28}$ ) compared to those who had mild or moderate stenosis ( $\leq 89 \%$ ECST). However, estimated risk of developing any ipsilateral ischemic cerebral events in a five-year period was estimated to be high in only $9 \%$ of the patients who had a stable carotid plaque despite $\geq 70 \%$ stenosis using the ECST criteria.

The implications of risk stratification found by the ACSRS collaborators and other prospective studies ${ }^{20,29}$ provide evidence that the vast majority of patients are better off with conservative treatment. Also, patients at higher risk could be identified and offered operative treatment. However, validation of predicted risk in that study was limited, since it was done for the same group of patients on whom the score was developed. These findings need to be validated in prospective studies using the best current medical therapy.

\section{Risk stratification}

Several factors have been associated with ipsilateral events to carotid plaque (Chart 1). The evidence for each of these factors is further discussed. 


\section{Degree of stenosis}

The degree of stenosis remains the mainstay criterion upon which vascular interventionists use to decide who should have a carotid artery intervention. The degree importance of the carotid stenosis and its correlation to cerebrovascular events have been demonstrated by several studies ${ }^{4,10,18,28}$. Treatment is often indicated for ACAD when a $>60 \%$ stenosis is detected ${ }^{4,5,28}$.

The shortcoming of relying only upon the degree of stenosis alone is that not all plaques that generate a vessel stenosis are unstable enough to prompt distal embolization or trigger an episode of in situ thrombosis, with subsequent occlusion of the vessel. Spence et al., investigating 319 ACAD patients with $>60 \%$ stenosis, demonstrated that $<1 \%$ of them developed stroke if there were no high intensity transient signals (microemboli) on transcranial Doppler (TCD) ${ }^{29}$. The ACSRS patients with moderate to severe stenosis had more cerebral ischemic events than those with $<50 \%$ stenosis. However, only $9 \%$ of patients with $\geq 70 \%$ stenosis were deemed to have high risk for cerebral or retinal events based on other risk factors, such as absence of contralateral TIA or stroke, no discrete white areas (DWAs), plaque area, and GSM. In addition, Kakkos et al., analyzing

Chart 1. Factors associated with ipsilateral events in patients bearing a carotid artery atherosclerotic plaque.

\begin{tabular}{|l|}
\hline Factors \\
\hline Degree of stenosis \\
Plaque morphology \\
Surface \\
Contents \\
Volume \\
Plaque motion \\
Contralateral ischemic brain attack \\
Baseline infarcts on CT/MR \\
Transcranial Doppler \\
\hline
\end{tabular}

CT - computed tomography; MR - magnetic resonance. data of 462 ACAD patients with 60 to $99 \%$ stenosis, showed that silent CT embolic infarcts have doubled the risk for developing a stroke compared to those without infarction ${ }^{30}$. Therefore, the degree of stenosis alone may no longer reflect or predict the group of patients who will have a stroke and may benefit from carotid artery intervention.

\section{Plaque morphology}

The study of plaque morphology includes the following: surface characteristics, echogenicity, distribution of plaque content, volume, and plaque kinetics. These have been studied by several methods such as DU, magnetic resonance image (MRI), CT, and histology. Prospective studies supporting the role of plaque morphology are displayed in Table $1^{9,12,13,19,31-35}$.

\section{Surface}

The surface of the plaque is defined as the linen that covers the plaque contents. It is defined as smooth, irregular, and ulcerated. Arteriographic and histological analysis provided by endarterectomy specimens have been utilized to detect surface abnormalities of the plaque ${ }^{26,36,37}$. Ulceration has often been characterized as a depression of $>1 \mathrm{~mm}$ in width and depth ${ }^{36,37}$; however, its real definition is loss of endothelial lining. Noninvasive imaging evaluation is more adequately used in ACAD reserving catheter-based angiogram for endovascular interventions and it is rarely for diagnostic purposes ${ }^{36,38-41}$.

The sensitivity and specificity of the DU on detecting ulceration have improved over the years ${ }^{36,38,39}$. Both B-mode imaging and flow disturbance originated by ulceration can be used to identify surface abnormalities on $\mathrm{DU}^{42-44}$. The improvement of ultrasound imaging and possibility of 3D format certainly will assist in increasing the likelihood of ulcer detection. A study of 84 carotid arteries using 3D ultrasound imaging

Table 1. Prospective studies supporting the role of plaque morphology on developing ipsilateral cerebrovascular events.

\begin{tabular}{|c|c|c|c|c|}
\hline Published studies & Year of publication & $\mathrm{n}$ & Follow-up, months (range) & Institution \\
\hline Johnson et al. ${ }^{12}$ & 1985 & 297 & 36 & Single-center \\
\hline Sterpetti et al. ${ }^{31}$ & 1988 & 214 & $34(26-39)$ & Single-center \\
\hline Langsfeld et al..$^{32}$ & 1989 & 289 & $22(3-48)$ & Single-center \\
\hline Bock et al. ${ }^{13}$ & 1993 & 242 & $28(n / a)$ & Single-center \\
\hline $\mathrm{CHS}^{33}$ & 1998 & 104 & $40(0.3-49)$ & Multi-center \\
\hline Tromsø study*34 & 2001 & 223 & 36 & Multi-center \\
\hline Nadareishvili et al. ${ }^{19}$ & 2002 & 106 & $120(60-216)$ & Multi-center \\
\hline Grønholdt et al. ${ }^{35}$ & 2002 & 246 & $53(36-70)$ & Multi-center \\
\hline ACSRS $^{9}$ & 2010 & 1121 & $48(6-96)$ & Multi-center \\
\hline
\end{tabular}

*297 carotid arteries in 297 patients were followed-up for three years; six died but it is not reported if other patients were lost; CHS - Cardiovascular Health Study; ACSRS - Asymptomatic Carotid Stenosis and Risk of Stroke (Study Group); **All patients were followed-up for 36 months. 
demonstrated higher detection rates of ulceration compared with $2 \mathrm{D}$ ultrasound ${ }^{42}$. A high-resolution MR imaging and a multi-detector CT angiography have also shown very good results of ulceration detection ${ }^{40,45}$.

The plaque ulceration might be included in the risk stratification due to its importance of generating embolic and thrombotic events. Frequently, surface abnormalities such as ulceration are found in symptomatic patients, but they can be found in up to $60 \%$ of the asymptomatic patients $s^{37,46,47}$. The risk of stroke in patients with carotid stenosis and ulcerated plaque was doubled among patients with $>70 \%$ stenosis compared to those without plaque ulceration $^{47}$. In addition, asymptomatic carotid artery plaques that are ulcerated or have surface thrombi were found to be associated with higher cerebral embolization rate up to $53 \%$ when stenotic plaques are present $t^{48,49}$.

Another important surface feature is the neovascularization and adventitial vasa vasorum of the plaque. Neovascularization of an atheroma was initially described in patients with coronary artery disease triggering myocardial ischemic events ${ }^{50,51}$. Investigation of the adventitial hyperplastic network in patients with carotid disease has also been performed using MR, CT, or DU with contrast ${ }^{52-54}$. The latter is of great interest in vascular practice due to wide availability, short learning curve and anatomic access due to relative superficial location of carotid artery. Vicenzini et al., studying 23 patients with ACAD, demonstrated that neovascularization is constantly present underneath ulcerated areas rendering the plaque potentially more vulnera$b^{2} e^{55}$. In addition, the angiogenesis network was identified coming from the adventitia towards the inner layers of the plaque, but this pattern was not detected in hyperechoic plaques with acoustic shadow (calcified) nor in those with advance inflammatory changes, such as hypoechoic necrotic plaques ${ }^{55}$. Regression of the adventitial vasa vasorum in a patient upon initiation of treatment with statins was reported suggesting a potential role for treatment monitoring ${ }^{56}$.

Plaque cap thickness has been correlated with plaque vulnerability. In a study of 22 patients, who underwent CEA, a greater number of plaques with cap thickness defined as $<200 \mu \mathrm{m}$ was found to be associated with cerebrovascular events $(\mathrm{p}=0.01)^{57}$. In another study, the histological analysis of 105 plaques in asymptomatic and symptomatic patients demonstrated that the presence of an echogenic cap was four times more frequent in the symptomatic ones than in those with asymptomatic plaques $(\mathrm{p}<0.05)^{58}$. In addition, the echolucent region juxtaluminal was more often present in symptomatic plaques $(67 v s .33 \% \text {; } \mathrm{p}<0.01)^{58}$.

\section{Plaque content and distribution}

The amount of lipid and its characteristic is variable and related to oxidative reactions led by inflammation, misbalanced production, and scavenging of free radicals. The vulnerability of the plaque is directly linked to the amount of core necrosis and intra-plaque hemorrhagic events ${ }^{37,59}$. Distinct nomenclature is already used to characterize the plaque morphology (i.e., echolucent $v s$. echogenic, soft $v s$ dense, complicated $v s$. uncomplicated $)^{12,60,61}$. Plaques have been classified in four or five types based on their echogenicity ${ }^{62}$. The morphologic classification and the corresponding ultrasound images is shown on Figure 1. In addition to the macroscopic ultrasonic evaluation of echogenicity, the GSM defined that a score is obtained using computer software as a surrogate marker of plaque vulnerability ${ }^{63,64}$. In fact, lipid-rich plaque core is seen in the DU as echolucent, and fibrotic components as echogenic.

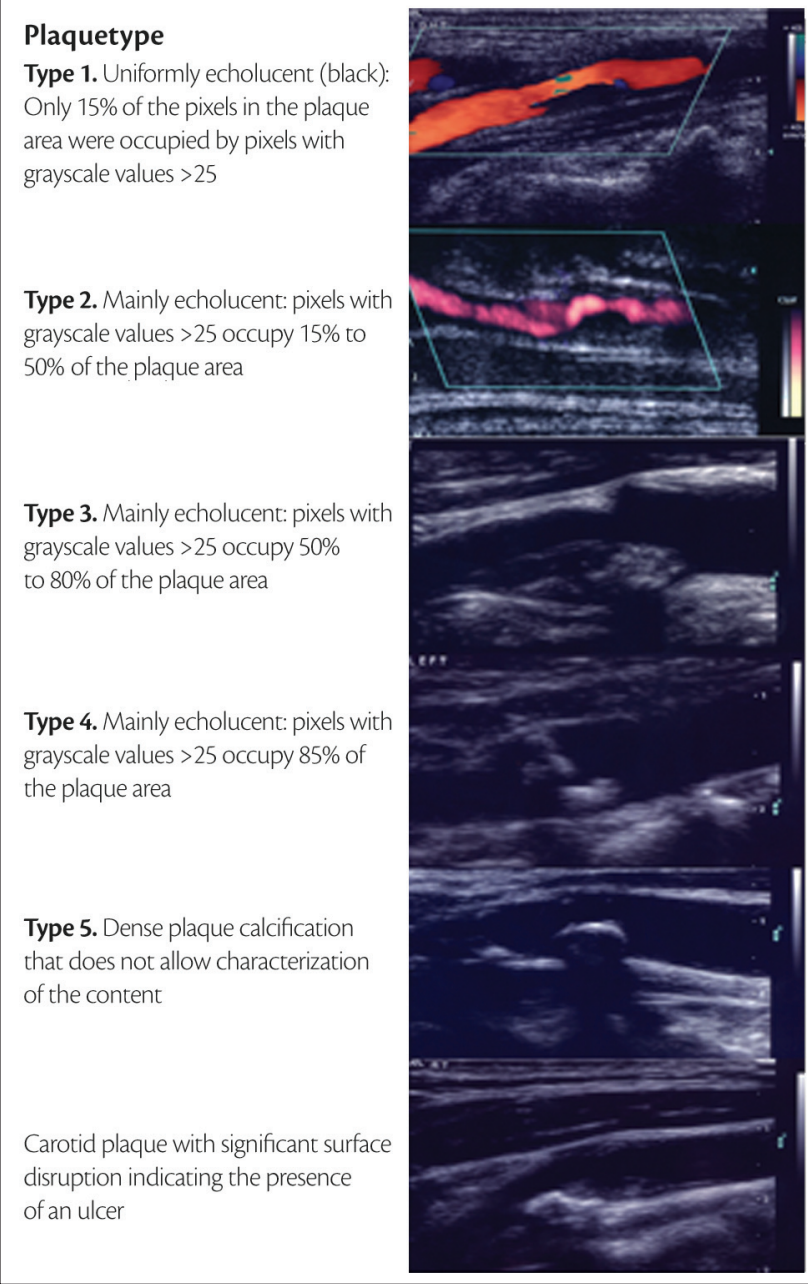

Figure 1. Carotid artery plaque morphology and its characteristics. 
Abu Rahma et al. demonstrated that plaque heterogeneity is the most important predictor of intra-plaque hemorrhage and symptoms related to cerebrovascular events ${ }^{60,61}$. The association of GSM and DWAs with the homogeneity of the plaque was also described by Nicolaides et al. in a study of 1,121 asymptomatic patients, which showed that plaques with DWAs and lower GSM $(<15)$ are more prone to generate cerebral and retinal events.

The association of macrophages and plaque lipid content and hemorrhage has been established ${ }^{65,66}$. In a study of 106 patients who had DU and subsequently underwent endarterectomy with plaque histology analysis, a higher concentration of macrophages in the plaque was related to more echolucent plaques ${ }^{65}$. The use of aspirin was associated with decreased number of macrophages and therefore inflammatory changes ${ }^{65}$. Interestingly, echolucent plaques are more commonly found in women than in men with carotid stenosis $>50 \%$ and triglyceride-rich lipoproteins ${ }^{66}$. Other blood lipid fraction misbalances, such as low levels of high-density lipoprotein (HDL), were also associated with echolucent plaques ${ }^{67}$.

In the Tromso study, 223 patients with carotid artery stenosis were compared to 215 age-gender matched control patients demonstrating that $28 \%$ who had stenotic plaques and predominantly or completely echolucent plaques had an ipsilateral cerebrovascular event ${ }^{34}$. By contrast, in the same study, only $4 \%$ of patients with predominantly echogenic or completely echogenic plaques had ipsilateral cerebrovascular events ${ }^{34}$.

\section{Volume}

Volume analysis can be achieved using ultrasound, CT or MRI. Frequently, the method used in CT and MRI is the demarcation of the regions of interest in multiple planes generating the area of the plaque that is added together creating a 3D image ${ }^{68-70}$. The ultrasonic assessment of the plaque volume is achieved using a real-time cine-loop recording application, which utilizes the sequential $2 \mathrm{D}$ gray scale imaging ${ }^{42}$.

The gender differences related to the plaque volume was evaluated in a study with 131 asymptomatic carotid plaques using a 3.0-Tesla, which showed that an increased volume of lipid-rich necrotic core (LRNC) is more prevalent in men ${ }^{70}$. Underhill et al., analyzing predictors of surface disruption and subsequent cerebral ischemic event also using MR in a cohort of 180 asymptomatic patients with carotid stenosis between 50 to $79 \%$, demonstrated that a larger percentage of LRNC volume (16.1 \pm 14.9 vs. $3.1 \pm 7.1 \%$, respectively; $\mathrm{p}<0.001)$ was associated with surface disruption ${ }^{59}$.

Corroborating to the fate of plaque disruption and its volume causing an ischemic cerebral event, a study of 100 acute symptomatic and asymptomatic plaques, based on MRI, found a larger plaque volume in asymptomatic plaques than in those that had a rupture. However, a larger core material was demonstrated in ruptured asymptomatic plaques than in the non-ruptured asymptomatic plaques perhaps signalizing to a pre-symptomatic state in patients with large volume plaques ${ }^{68}$. Conversely, Nandalur et al., analyzing the correlation of plaque volume utilizing MDCTA among 102 carotid plaques (35 symptomatic - stenosis: $82.0 \pm 11.9 \%$ ) and 67 asymptomatic patients (stenosis: $79.4 \pm 10.8 \%$ ), demonstrated that the absolute volume of the plaque does not correlate with ischemic cerebral events, but the amount of calcification found in the CT does ${ }^{69}$.

The use of $3 \mathrm{D}$ ultrasound measurement of plaque volume and its changes related to medical treatment has been described $^{71}$. In a placebo-controlled study, 17 patients taking $80 \mathrm{mg}$ daily atorvastatin were compared to 21 patients in the placebo arm demonstrating plaque volume reduction in the Statin Group versus plaque progression in the Placebo Group $^{71}$. These initial findings signalize to a potential tool for treatment monitoring and surveillance in asymptomatic patients.

The actual limitations of the plaque volume studies are the relative small number of studies with small number of patients, a variety of measuring methods, and poor longterm prospective follow-up to define the cutoffs to assist in selecting patients at risk of an ischemic cerebral event.

\section{Plaque motion}

Some experimental and clinical observations of plaque motion have been made $e^{72-76}$. The rationale of plaque motion relies on the constant displacement vectors applied to the plaque surface by the dragging forces generated by the blood stream during the cardiac cycle.

A proposition of changing in plaque motion following medical treatment was done by Lenzi and Vicenzini. In the evaluation of two asymptomatic patients, the carotid plaque and vessel wall move in the opposite direction of the blood flow $^{72}$. The motion pattern described was hypothesized to be a potential cause of external plaque layer disruption creating a thrombogenic surface with exposure of the plaque material and embolization of plaque fragments.

The differences of the plaque surface motion were assessed in asymptomatic $(n=18)$ and symptomatic $(n=13)$, 
demonstrating maximal discrepant surface velocity higher in symptomatic patients than in those asymptomatic mainly in the cardiac systole ${ }^{73}$. In addition, asymptomatic patients were found to have more homogenous orientation and magnitude of velocity vectors ${ }^{73}$. Heterogeneous plaque motion pattern was also found inside the plaque with some regions fairly immobile and others highly mobile, in a study performed in 11 volunteers by Bang and colleagues ${ }^{76}$.

Clinical association of plaque motion was assessed in a study of 242 stroke and 336 TIA patients showing that the presence of longitudinal plaque motion tripled the risk of developing a TIA $(p=0.2)^{74}$. Regardless some evidence and delineation of the plaque kinetics underpinnings, it remains an evolving concept that need more clinical grounding assessments, but certainly will be added to the risk stratification of ACAD patients in the future.

\section{Contralateral ischemic brain attack}

The association of contralateral symptomatic carotid stenosis with an ipsilateral asymptomatic carotid stenosis was demonstrated in the ACST ${ }^{5}$. In this trial, patients with contralateral ischemic brain events secondary to carotid plaque were found to have more ipsilateral strokes than those with ipsilateral carotid stenosis and no significant or asymptomatic contralateral diseases (35 vs. 11, $\mathrm{p}=0.0004)^{5}$.

The results of the ACST are in agreement with those found in the ACSRS trial conducted by Nicolaides et al. ${ }^{10}$. In the latter, 1,121 patients with asymptomatic carotid plaque who had a history of contralateral TIAs or stroke had an increased risk of ipsilateral cerebrovascular or retinal ischemic event (hazard ratio $-\mathrm{HR}=2.35$; 95\%CI 1.60-3.43) and ipsilateral stroke $(\mathrm{HR}=3.03 \text {; 95\%CI } 1.77-5.20)^{10}$.

\section{Baseline Infarcts On CT Scan/MRI}

The embolic activity of carotid plaque can be indirectly stated in the absence of cardioembolism by finding a silent brain infarct in a CT or MRI. A reasonable number of patients with high-grade asymptomatic carotid stenosis will develop a silent embolic infarct, which may perhaps be predictive of a future TIA or stroke ${ }^{30}$.

In a large prospective multicenter study of 821 patients, $17 \%$ had a CT scan showing cortical, subcortical, and basal ganglia brain infarcts in patients with $\geq 60 \%$ asymptomatic carotid artery stenosis. In that study the cumulative strokefree rate was 0.92 (1.0\% annual stroke rate) in the absence of embolic infarcts and 0.71 (3.6\% annual stroke rate) in their presence $(\mathrm{p}<0.002 ; \mathrm{HR}=3.0 ; 95 \% \mathrm{CI} 1.46-6.29)^{30}$. The relationship between silent brain infarcts and type of plaque has also been proposed ${ }^{77,78}$. Sabetai et al., analyzing the echo-texture of 273 asymptomatic carotid artery plaques, demonstrated that echolucent plaques with low GSM were associated with significantly more silent non-lacunar brain infarcts than those with high GSM ${ }^{77}$. Tegos and colleagues investigating 419 carotid plaques found silent CT brain infarcts in $10 \%$ of asymptomatic patients, being the subcortical and cortical infarcts encountered more in those bearing hypoechogenic plaques ${ }^{78}$.

The findings of silent brain infarcts in patients combined to other factors herein discussed may assist in selecting high-risk asymptomatic patients for treatment. Evaluation with CT or MRI during follow-up of asymptomatic patients is warranted.

TCD

TCD has been described as an important tool in the assessment of patients with $\mathrm{ACAD}^{29,79-81}$. The evaluation of cerebral vasoreactivity response and identification of plaque fragments traveling through the brain circulation can be done by the TCD. The embolic debris originated from a carotid artery plaque can be detected as embolic signals insonating the middle cerebral or ophthalmic artery ${ }^{29}$. The cerebral vasoreactivity is tested asking the patients to inspire $6 \%$ carbon dioxide or by injecting acetazolamide to estimate the vasodilator effects in the cerebral blood flow ${ }^{82,83}$.

The Asymptomatic Carotid Emboli Study (ACES), a prospective observational study that recruited 482 patients to assess the embolic activity of carotid plaques, demonstrated that about $16 \%$ of the patients had a positive TCD signal compatible with plaque fragments embolization $^{80}$. In those who had emboli signals, the risk of ipsilateral stroke and TIA was higher compared to those without emboli signals $(\mathrm{HR}=2.54,95 \% \mathrm{CI} 1.20-5.36 ; \mathrm{p}=0.015)$ as well as the risk of ipsilateral stroke alone $(\mathrm{HR}=5.57$, 95\%CI 1.61-19.32; $\mathrm{p}=0.007)^{80}$. Notably, in a Scandinavian prospective cohort study of 62 patients with ACAD the incidence of emboli signals in patients who had a TIA was $16 \%$. Stroke rate in this cohort was higher in those with positive emboli signal than in the ones with no emboli signals by TCD (30 vs. $2 \%$; $\mathrm{p}<0.05)$.

Spence et al., following a cohort of 319 for a two-year period, reported incidence of $10 \%$ of patients with ACAD with positive embolic signals. Those patients with negative TCD for embolic signals had lower risk of stroke during the first year of follow-up $(15.6 \%, 95 \%$ CI $4.1-79$ vs. $1 \%$, 95\%CI 
1.01-1.36; $\mathrm{p}<0.0001)$. As previously stated the risk of stroke was nearly $1 \%$ in asymptomatic patients, which raised concerns about the higher than $1 \%$ risk of treating the plaque with either endarterectomy or stenting. Remarkably, in a subsequent prospective study of 468 patients, the same authors demonstrated the trends of embolic signals rate throughout a seven-year period showing decreasing rates of embolization due to likely stabilization of the plaque secondary to an expanding number of patients on best medical therapy ${ }^{79}$.

A subset analysis of the 106 patients participating in the ACES found that there was no association between impaired cerebral vasoreactivity and recurrent ischemic events, but the study was unpowered by small number of patients; however, a meta-analysis performed by the authors showed association between impaired vasoreactivity and future ischemic cerebral events ${ }^{83}$. In reality, the ACES collaborators advocated that vasoreactivity measurement will require further data to be supported as a diagnostic tool in the risk stratification algorithm in patients with ACAD.

\section{Limitations}

There is level I evidence based upon large trials to support a carotid intervention in patients with ACAD. However, only $1 \%$ of patients with ACAD will have a stroke and only about $6 \%$ of interventions in asymptomatic patients may be justifiable. In addition, the annual risk of stroke is in decline, as opposed to the stroke rate in 1995, when the vast majority of patients were not on BMT. Perhaps, the standard of care on which that level I evidence is based has changed over the past 15 years.

Using low-dose angiotensin-converting enzyme inhibition, aggressive blood pressure control in type II diabetes mellitus, multi-agent antihypertensive therapy, aggressive lifestyle modification, aspirin and statins, and discontinuation of hormonal therapy in females are all available approaches in medical therapy. However, drawbacks of some studies that advocate BMT alone as the preferred treatment include small sample size, inconsistent inclusion criterion when considering the current Doppler ultrasound velocity cutoff for high-grade stenosis (i.e. PSV $>150 \mathrm{~cm} / \mathrm{s}$ instead of $230 \mathrm{~cm} / \mathrm{s}$ ), debatable endpoints, crossovers and shortterm follow-up. Thus, a study arm utilizing the current standards for BMT is still required in larger ACAD trials to gauge the real impact of BMT alone vs BMT plus carotid intervention.

Other limitations of several ACAD studies are the simplistic use of stenosis degree rather than employing TCD, silent brain infarct, or plaque morphology. More powered randomized prospective trials evaluating the predictive value of TCD, baseline ipsilateral infarcts on CT scan and plaque morphology are still warranted in order to identify 'high risk for stroke' patients, who will benefit from an intervention plus BMT rather than BMT alone.

\section{Conclusions}

Asymptomatic carotid artery stenosis risk stratification remains a complex and evolving subject in Vascular Surgery. Mass indication for carotid interventions will not reduce the burden of stroke in $>90 \%$ of patients with ACAD and therefore are ultimately unnecessary. The degree of carotid plaque stenosis in asymptomatic patients as the only criterion to indicate an enormous number of interventions has been put in dispute. Carotid plaque characteristics, such as type of the plaque, plaque surface and volume, history of contralateral TIAs and stroke, evaluation of silent embolic strokes by CT or MRI and assessment of embolic particles by TCD must be combined at the final patient assessment, whenever available, in order to offer the best treatment tailored to each patient specificities.

\section{References}

1. Qureshi Al, Suri MF, Nasar A, et al. Changes in cost and outcome among US patients with stroke hospitalized in 1990 to 1991 and those hospitalized in 2000 to 2001. Stroke. 2007;38(7):2180-4.

2. Reed SD, Blough DK, Meyer K, Jarvik JG. Inpatient costs, length of stay, and mortality for cerebrovascular events in community hospitals. Neurology. 2001;57(2):305-14.

3. Cabral NL, Goncalves AR, Longo AL, et al. Trends in stroke incidence, mortality and case fatality rates in Joinville, Brazil: 1995-2006. J Neurol Neurosurg Psychiatry. 2009;80(7):749-54.

4. Endarterectomy for asymptomatic carotid artery stenosis. Executive Committee for the Asymptomatic Carotid Atherosclerosis Study. JAMA. 1995;273(18):1421-8.

5. Halliday A, Mansfield A, Marro J, et al. Prevention of disabling and fatal strokes by successful carotid endarterectomy in patients without recent neurological symptoms: randomised controlled trial. Lancet. 2004;363(9420):1491-502.

6. Abbott AL. Medical (nonsurgical) intervention alone is now best for prevention of stroke associated with asymptomatic severe carotid stenosis: results of a systematic review and analysis. Stroke. 2009;40(10):e573-83.

7. Marquardt L, Geraghty OC, Mehta Z, Rothwell PM. Low risk of ipsilateral stroke in patients with asymptomatic carotid stenosis on best medical treatment: a prospective, population-based study. Stroke. 2010;41(1):e11-7.

8. Silver FL, Mackey A, Clark WM, et al. Safety of stenting and endarterectomy by symptomatic status in the Carotid Revascularization Endarterectomy Versus Stenting Trial (CREST). Stroke. 2011; $42(3): 675-80$ 
9. Rothwell PM, Warlow CP. Low risk of ischemic stroke in patients with reduced internal carotid artery lumen diameter distal to severe symptomatic carotid stenosis: cerebral protection due to low poststenotic flow? On behalf of the European Carotid Surgery Trialists' Collaborative Group. Stroke. 2000;31(3):622-30.

10. Nicolaides AN, Kakkos SK, Kyriacou E, et al. Asymptomatic internal carotid artery stenosis and cerebrovascular risk stratification. J Vasc Surg. 2010;52(6):1486-96.

11. Kakkos SK, Nicolaides AN, Kyriacou E, et al. Computerized texture analysis of carotid plaque ultrasonic images can identify unstable plaques associated with ipsilateral neurological symptoms. Angiology. 2011;62(4):317-28.

12. Johnson JM, Kennelly MM, Decesare D, Morgan S, Sparrow A. Natural history of asymptomatic carotid plaque. Arch Surg. 1985;120(9):1010-2.

13. Bock RW, Gray-Weale AC, Mock PA, et al. The natural history of asymptomatic carotid artery disease. J Vasc Surg. 1993;17(1):160-9.

14. Chambers BR, Norris JW. Outcome in patients with asymptomatic neck bruits. N Engl J Med. 1986;315(14):860-5.

15. Hennerici M, Hulsbomer HB, Hefter H, Lammerts D, Rautenberg W. Natural history of asymptomatic extracranial arterial disease. Results of a long-term prospective study. Brain. 1987;110 (Pt 3):777-91.

16. Norris JW, Zhu CZ, Bornstein NM, Chambers BR. Vascular risks of asymptomatic carotid stenosis. Stroke. 1991;22(12):1485-90.

17. Zhu CZ, Norris JW. A therapeutic window for carotid endarterectomy in patients with asymptomatic carotid stenosis. Can J Surg. 1991;34(5):437-40

18. Mackey AE, Abrahamowicz M, Langlois $Y$, et al. Outcome of asymptomatic patients with carotid disease. Asymptomatic Cervical Bruit Study Group. Neurology. 1997;48(4):896-903.

19. Nadareishvili ZG, Rothwell PM, Beletsky V, Pagniello A, Norris JW. Long-term risk of stroke and other vascular events in patients with asymptomatic carotid artery stenosis. Arch Neurol. 2002;59(7):1162-6.

20. Goessens BM, Visseren FL, Kappelle LJ, Algra A, van der Graaf Y. Asymptomatic carotid artery stenosis and the risk of new vascular events in patients with manifest arterial disease: the SMART study. Stroke. 2007;38(5):1470-5.

21. Huber TS, Wheeler KG, Cuddeback JK, Dame DA, Flynn TC, Seeger JM. Effect of the Asymptomatic Carotid Atherosclerosis Study on carotid endarterectomy in Florida. Stroke. 1998;29(6):1099-105.

22. Huber TS, Durance PW, Kazmers A, Jacobs LA. Effect of the Asymptomatic Carotid Atherosclerosis Study on carotid endarterectomy in Veterans Affairs medical centers. Arch Surg. 1997;132(10):1134-9.

23. Woo K, Garg J, Hye RJ, Dilley RB. Contemporary results of carotid endarterectomy for asymptomatic carotid stenosis. Stroke. 2010;41(5):975-9.

24. Karp HR, Flanders WD, Shipp CC, Taylor B, Martin D. Carotid endarterectomy among Medicare beneficiaries: a statewide evaluation of appropriateness and outcome. Stroke. 1998;29(1):46-52.
25. Naylor AR, Gaines PA, Rothwell PM. Who benefits most from intervention for asymptomatic carotid stenosis: patients or professionals? Eur I Vasc Endovasc Surg. 2009;37(6):625-32.

26. Moore WS, Boren C, Malone JM, et al. Natural history of nonstenotic, asymptomatic ulcerative lesions of the carotid artery. Arch Surg. 1978;113(11):1352-9.

27. Geroulakos G, Hobson RW, Nicolaides A. Ultrasonographic carotid plaque morphology in predicting stroke risk. Br J Surg. 1996;83(5):582-7.

28. MRC European Carotid Surgery Trial: interim results for symptomatic patients with severe (70-99\%) or with mild (0-29\%) carotid stenosis. European Carotid Surgery Trialists' Collaborative Group. Lancet. 1991;337(8752):1235-43.

29. Spence JD, Tamayo A, Lownie SP, Ng WP, Ferguson GG. Absence of microemboli on transcranial Doppler identifies low-risk patients with asymptomatic carotid stenosis. Stroke. 2005;36(11):2373-8.

30. Kakkos SK, Sabetai M, Tegos T, et al. Silent embolic infarcts on computed tomography brain scans and risk of ipsilateral hemispheric events in patients with asymptomatic internal carotid artery stenosis. J Vasc Surg. 2009;49(4):902-9.

31. Sterpetti AV, Schultz RD, Feldhaus RJ. Asymptomatic carotid artery stenosis on the side contralateral to endarterectomy. A comparison between patients with and those without operation. J Vasc Surg. 1988;8(4):453-9.

32. Langsfeld M, Gray-Weale AC, Lusby RJ. The role of plaque morphology and diameter reduction in the development of new symptoms in asymptomatic carotid arteries. J Vasc Surg. 1989;9(4):548-57.

33. Polak JF, Shemanski L, O'Leary DH, et al. Hypoechoic plaque at US of the carotid artery: an independent risk factor for incident stroke in adults aged 65 years or older. Cardiovascular Health Study. Radiology. 1998;208(3):649-54.

34. Mathiesen EB, Bonaa KH, Joakimsen O. Echolucent plaques are associated with high risk of ischemic cerebrovascular events in carotid stenosis: the tromso study. Circulation. 2001;103(17):2171-5.

35. Gronholdt ML, Nordestgaard BG, Schroeder TV, Vorstrup S, Sillesen $\mathrm{H}$. Ultrasonic echolucent carotid plaques predict future strokes. Circulation. 2001;104(1):68-73.

36. O'Leary DH, Holen J, Ricotta J), Roe S, Schenk EA. Carotid bifurcation disease: prediction of ulceration with B-mode US. Radiology. 1987;162(2):523-5.

37. Park AE, MCCarthy WJ, Pearce WH, Matsumura JS, Yao JS. Carotid plaque morphology correlates with presenting symptomatology. J Vasc Surg. 1998;27(5):872-8.

38. O'Donnell TFJr., Erdoes L, Mackey WC, et al. Correlation ofB-mode ultrasound imaging and arteriography with pathologic findings at carotid endarterectomy. Arch Surg. 1985;120(4):443-9.

39. Comerota AJ, Katz ML, White JV, Grosh JD. The preoperative diagnosis of the ulcerated carotid atheroma.J Vasc Surg. 1990;11(4):50510.

40. Tartari S, Rizzati R, Righi R, et al. High-Resolution MRI of Carotid Plaque With a Neurovascular Coil and Contrast-Enhanced MR Angiography: One-Stop Shopping for the Comprehensive Assessment of Carotid Atherosclerosis. Am J Roentgenol. 2011;196(5):1164-71. 
41. Homburg PJ, Plas G), Rozie S, van der Lugt A, Dippel DW. Prevalence and calcification of intracranial arterial stenotic lesions as assessed with multidetector computed tomography angiography. Stroke. 2011;42(5):1244-50.

42. Heliopoulos J, Vadikolias K, Piperidou C, Mitsias P. Detection of carotid artery plaque ulceration using 3-dimensional ultrasound. J Neuroimaging. 2011;21(2):126-31.

43. Wong EY, Nikolov HN, Thorne ML, Poepping TL, Rankin RN, Holdsworth DW. Clinical Doppler ultrasound for the assessment of plaque ulceration in the stenosed carotid bifurcation by detection of distal turbulence intensity: a matched model study. Eur Radiol. 2009;19(11):2739-49.

44. Sitzer M, Muller W, Rademacher J, et al. Color-flow Dopplerassisted duplex imaging fails to detect ulceration in high-grade internal carotid artery stenosis. J Vasc Surg. 1996;23(3):461-5.

45. Homburg PJ, Rozie S, van Gils MJ, et al. Association between carotid artery plaque ulceration and plaque composition evaluated with multidetector CT angiography. Stroke. 2011;42(2):367-72.

46. Fisher M, Paganini-Hill A, Martin A, et al. Carotid plaque pathology: thrombosis, ulceration, and stroke pathogenesis. Stroke. 2005;36(2):253-7.

47. Eliasziw M, Streifler JY, Fox AJ, Hachinski VC, Ferguson GG, Barnett HJ. Significance of plaque ulceration in symptomatic patients with high-grade carotid stenosis. North American Symptomatic Carotid Endarterectomy Trial. Stroke. 1994;25(2):304-8.

48. Bassiouny HS, Davis H, Massawa N, Gewertz BL, Glagov S, Zarins CK. Critical carotid stenoses: morphologic and chemical similarity between symptomatic and asymptomatic plaques. J Vasc Surg. 1989;9(2):202-12.

49. Sitzer M, Muller W, Siebler $M$, et al. Plaque ulceration and lumen thrombus are the main sources of cerebral microemboli in highgrade internal carotid artery stenosis. Stroke. 1995;26(7):1231-3.

50. Barger AC, Beeuwkes R 3rd. Rupture of coronary vasa vasorum as a trigger of acute myocardial infarction. Am J Cardiol. 1990;66(16):41G-3.

51. Kumamoto $M$, Nakashima $Y$, Sueishi K. Intimal neovascularization in human coronary atherosclerosis: its origin and pathophysiological significance. Hum Pathol. 1995;26(4):450-6.

52. Fuster V, Fayad ZA, Moreno PR, Poon M, Corti R, Badimon JJ. Atherothrombosis and high-risk plaque: Part II: approaches by noninvasive computed tomographic/magnetic resonance imaging. I Am Coll Cardiol. 2005;46(7):1209-18.

53. Fuster $\mathrm{V}$. The evolving role of $C T$ and MRI in atherothrombotic evaluation and management. Nat Clin Pract Cardiovasc Med. 2005;2(7):323.

54. Kerwin W, Hooker A, Spilker M, et al. Quantitative magnetic resonance imaging analysis of neovasculature volume in carotid atherosclerotic plaque. Circulation. 2003;107(6):851-6.

55. Vicenzini E, Giannoni MF, Puccinelli F, et al. Detection of carotid adventitial vasa vasorum and plaque vascularization with ultrasound cadence contrast pulse sequencing technique and echocontrast agent. Stroke. 2007;38(10):2841-3.

56. Feinstein SB. Contrast ultrasound imaging of the carotid artery vasa vasorum and atherosclerotic plaque neovascularization. J Am Coll Cardiol. 2006;48(2):236-43.
57. Faggioli GL, Pini R, Mauro R, et al. Identification of carotid 'vulnerable plaque' by contrast-enhanced ultrasonography: correlation with plaque histology, symptoms and cerebral computed tomography. Eur J Vasc Endovasc Surg. 2011;41(2):238-48.

58. Pedro LM, Pedro MM, Goncalves I, et al. Computer-assisted carotid plaque analysis: characteristics of plaques associated with cerebrovascular symptoms and cerebral infarction. Eur I Vasc Endovasc Surg. 2000;19(2):118-23.

59. Underhill HR, Yuan C, Yarnykh VL, et al. Predictors of surface disruption with MR imaging in asymptomatic carotid artery stenosis. Am J Neuroradiol. 2010;31(3):487-93.

60. AbuRahma AF, Wulu JT Jr., Crotty B. Carotid plaque ultrasonic heterogeneity and severity of stenosis. Stroke. 2002;33(7):1772-5.

61. AbuRahma AF, Kyer PD 3rd, Robinson PA, Hannay RS. The correlation of ultrasonic carotid plaque morphology and carotid plaque hemorrhage: clinical implications. Surgery. 1998;124(4):721-6.

62. Geroulakos G, Ramaswami G, Nicolaides A, et al. Characterization of symptomatic and asymptomatic carotid plaques using high-resolution real-time ultrasonography. $\mathrm{Br}$ J Surg. 1993;80(10):1274-7.

63. Griffin M, Nicolaides A, Kyriacou E. Normalisation of ultrasonic images of atherosclerotic plaques and reproducibility of grey scale median using dedicated software. Int Angiol. 2007;26(4):372-7.

64. Sabetai MM, Tegos TJ, Nicolaides AN, Dhanjil S, Pare G), Stevens JM. Reproducibility of computer-quantified carotid plaque echogenicity: can we overcome the subjectivity? Stroke. 2000;31(9):2189-96.

65. Gronholdt ML, Nordestgaard BG, Bentzon J, et al. Macrophages are associated with lipid-rich carotid artery plaques, echolucency on B-mode imaging, and elevated plasma lipid levels. J Vasc Surg. 2002;35(1):137-45.

66. Kofoed SC, Gronholdt ML, Bismuth J, Wilhjelm JE, Sillesen H, Nordestgaard BG. Echolucent, rupture-prone carotid plaques associated with elevated triglyceride-rich lipoproteins, particularly in women. J Vasc Surg. 2002;36(4):783-92.

67. Mathiesen EB, Bonaa KH, Joakimsen O. Low levels of high-density lipoprotein cholesterol are associated with echolucent carotid artery plaques: the tromso study. Stroke. 2001;32(9):1960-5.

68. Sadat U, Teng Z, Young VE, Graves MJ, GillardJH. Three-dimensional volumetric analysis of atherosclerotic plaques: a magnetic resonance imaging-based study of patients with moderate stenosis carotid artery disease. Int J Cardiovasc Imaging. 2010;26(8):897-904.

69. Nandalur KR, Hardie AD, Raghavan P, Schipper MJ, Baskurt E, Kramer CM. Composition of the stable carotid plaque: insights from a multidetector computed tomography study of plaque volume. Stroke. 2007;38(3):935-40.

70. Ota H, Reeves MJ, Zhu DC, et al. Sex differences in patients with asymptomatic carotid atherosclerotic plaque: in vivo 3.0-T magnetic resonance study. Stroke. 2010;41(8):1630-5.

71. Ainsworth CD, Blake CC, Tamayo A, Beletsky V, Fenster A, Spence JD. 3D ultrasound measurement of change in carotid plaque volume: a tool for rapid evaluation of new therapies. Stroke. 2005;36(9):1904-9.

72. Lenzi GL, Vicenzini $E$. The ruler is dead: an analysis of carotid plaque motion. Cerebrovasc Dis. 2007;23(2-3):121-5. 
73. Meairs S, Hennerici M. Four-dimensional ultrasonographic characterization of plaque surface motion in patients with symptomatic and asymptomatic carotid artery stenosis. Stroke. 1999;30(9):1807-13.

74. lannuzzi A, Wilcosky T, Mercuri M, Rubba P, Bryan FA, Bond MG. Ultrasonographic correlates of carotid atherosclerosis in transient ischemic attack and stroke. Stroke. 1995;26(4):614-9.

75. Chan KL. Two approaches to motion analysis of the ultrasound image sequence of carotid atheromatous plaque. Ultrasonics. 1993;31(2):117-23.

76. Bang J, Dahl T, Bruinsma A, Kaspersen JH, Nagelhus Hernes TA, Myhre $\mathrm{HO}$. A new method for analysis of motion of carotid plaques from RF ultrasound images. Ultrasound Med Biol. 2003;29(7):967-76.

77. Sabetai MM, Tegos T], Clifford C, et al. Carotid plaque echogenicity and types of silent CT-brain infarcts. Is there an association in patients with asymptomatic carotid stenosis? Int Angiol. 2001;20(1):51-7.

78. Tegos TJ, Sabetai MM, Nicolaides AN, Elatrozy TS, Dhanjil S, Stevens JM. Patterns of brain computed tomography infarction and carotid plaque echogenicity. J Vasc Surg. 2001;33(2): 334-9.

79. Spence JD, Coates V, Li H, et al. Effects of intensive medical therapy on microemboli and cardiovascular risk in asymptomatic carotid stenosis. Arch Neurol. 2010;67(2):180-6.

80. Markus HS, King A, Shipley M, et al. Asymptomatic embolisation for prediction of stroke in the Asymptomatic Carotid Emboli Study (ACES): a prospective observational study. Lancet Neurol. 2010;9(7):663-71.
81. Zhang C, Qu S, Li H, et al. Microembolic signals and carotid plaque characteristics in patients with asymptomatic carotid stenosis. Scand Cardiovasc J. 2009;43(5):345-51.

82. Lucertini G, Ermirio D, Belardi P. Cerebral haemodynamic aspects of severe carotid stenosis: asymptomatic vs symptomatic. Eur J Vasc Endovasc Surg. 2002;24(1):59-62.

83. King A, Serena J, Bornstein NM, Markus HS. Does impaired cerebrovascular reactivity predict stroke risk in asymptomatic carotid stenosis?: a prospective substudy of the asymptomatic carotid emboli study. Stroke. 2011;42(6):1550-5.

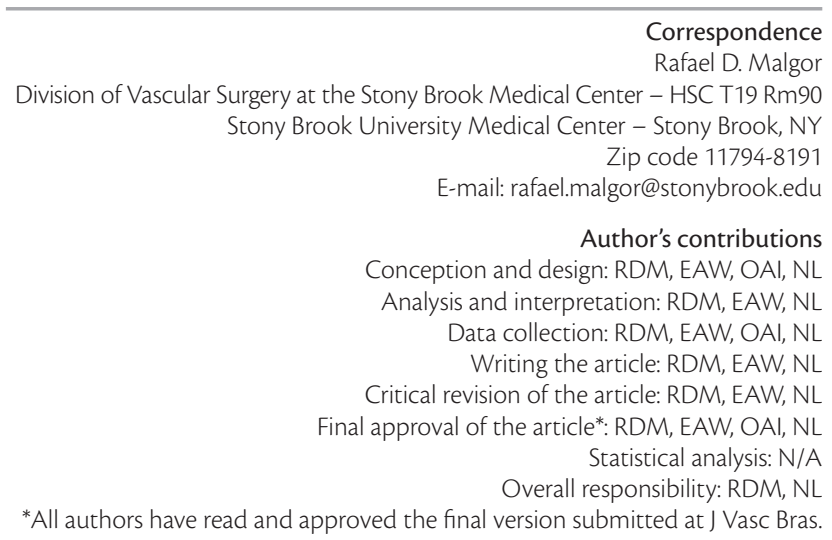

